\title{
Compliant Control of a Hydraulic Humanoid Joint
}

\author{
Darrin C. Bentivegna*†, Christopher G. Atkeson*†, and Jung-Yup Kim ${ }^{\dagger}$ \\ *ATR Computational Neuroscience Laboratories, \\ Department of Humanoid Robotics and Computational Neuroscience, Kyoto, Japan \\ ${ }^{\dagger}$ Carnegie Mellon University, Robotics Institute, Pittsburgh, PA, USA \\ (darrin@atr.jp, cga@cmu.edu, jungyupkim76@gmail.com)
}

\begin{abstract}
This paper presents an analysis of a hydraulic joint on a humanoid robot. Various controllers have been designed that allow the limb to have a range of characteristics such as being stiff or compliant.
\end{abstract}

\section{INTRODUCTION}

A humanoid robot needs to have limbs that satisfy a variety of criteria. While the robot is walking, the stance leg needs to be strong to support the entire weight of the robot. The swing leg needs to be compliant to account for any disturbances that might occur while the leg is moving into position and when it hits the ground. In double support, both legs need to be compliant to minimize internal forces. The characteristics of a leg will need to quickly change as the swing leg becomes the stance leg and the stance leg becomes the swing leg. The arms have similar constraints in that they may be tasked with carrying heavy objects, accurately picking up and placing objects, and softly interacting with humans. It is challenging to find an actuation mechanism that meets these criteria. Researchers are investigating a variety of actuator and controller configurations to create joints and limbs that can be both strong and compliant [1], [2], [3], [4]. There is also research into changing the gains of the controller while the device is operating to achieve a desired performance [5], [6]. Hyon et. al. used torque control loops for all joints of a hydraulically actuated humanoid robot [7], [8]. They demonstrated stable control, gravity compensation, and balancing of the entire robot. We will present an in-depth analysis of a single hydraulic joint. Other research on torque controlled hydraulic actuators include [9] and [10].

We are investigating the use of hydraulic actuators to provide the strength, speed, and compliance needed for a humanoid robot. Traditionally hydraulic actuators do not have the characteristics needed for compliant control [11]. The research presented here shows that our hydraulically actuated joints can achieve all these characteristics. Our Sarcos Primus System Humanoid Robot, Figure 1, consists of 51 actuated joints. The four joints that control eye pan and tilt and the single joint that controls mouth movement use electric servo motors. There are 12 joints that control hand movement and use pneumatic actuators. The other 34 joints use linear hydraulic actuators.

This paper presents our analysis of the hip pitch joint. The hip pitch and knee joints are particularly challenging for two reasons. First it is these joints that are mostly used for walking

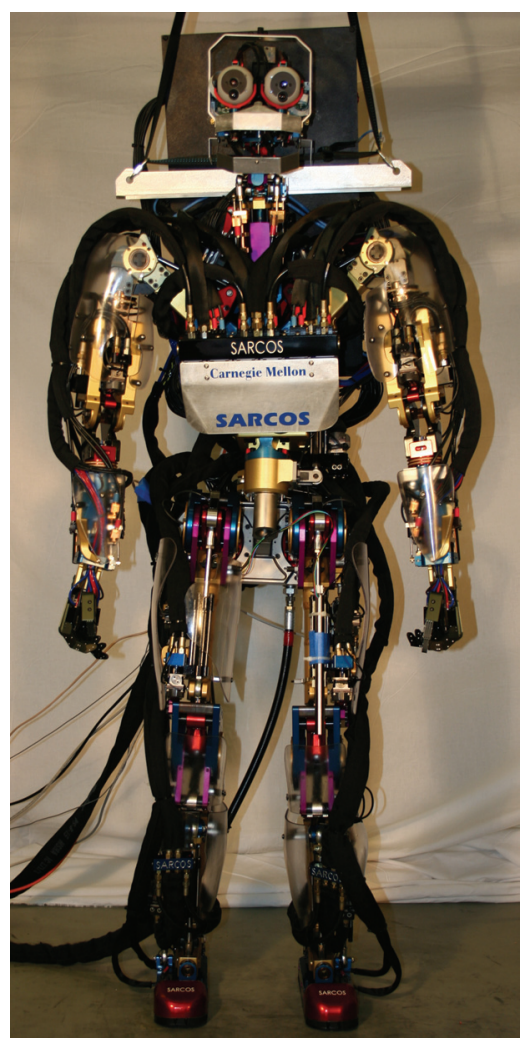

Fig. 1. The Sarcos Primus System Humanoid Robot.

and are subject to a wide variety of constraints as the leg changes from stance to swing. Second, these joints contain a proprietary linkage mechanism and a custom force sensor built into the linkage. Section II discusses this further and presents a model of experimental data.

We will demonstrate that for a single hydraulic joint we can achieve good torque control. This work is part of an ongoing effort to create controllers for all the joints of this robot. We expect future controllers will be more complicated in that they will be responsible for controlling multiple joints simultaneously. Therefore it is very important that we first accurately calibrate and understand individual joint operation before we delve into more complicated controllers. 


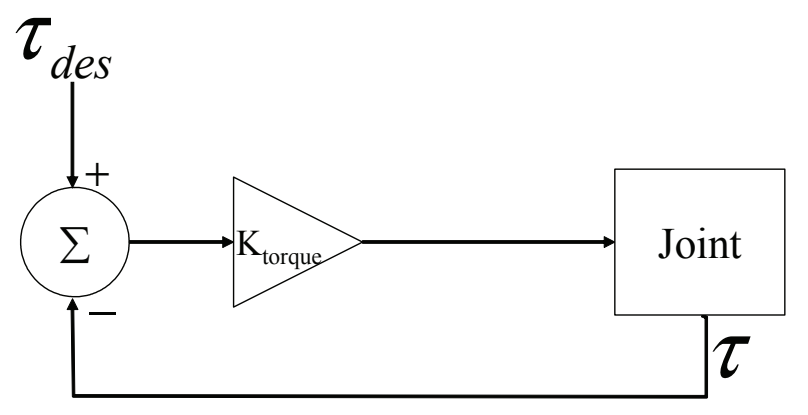

Fig. 2. Torque controller.

\section{HIP PITCH JOINT}

The hydraulically controlled joints use Moog flow control valves to control the pressure on opposite sides of a piston. For the hip pitch joint, the model number of the Moog valve is "MOOG 30-400A C03547-002", the diameter of the piston is $158.75 \mathrm{~mm}$, and the diameter of the rod is $95.25 \mathrm{~mm}$. The pressure of the oil is $3000 \mathrm{psi}$ for all results presented in this paper and is the normal operating pressure for the robot. The Moog valve is controlled by current and a current other than 0 will cause a differential oil flow to be applied to the opposing sides of the piston. In the case of no load on the actuator, the linear velocity of the actuator is proportional to the current.

The joints contain load cells, from which a torque can be computed, and are capable of running high gain torque control servos using the controller shown in Figure 2. An error signal, the difference between the desired torque, $\tau_{\text {des }}$, that the actual torque, $\tau$, is multiplied by the gain $K_{\text {torque }}$ to generate a valve command. The valve command controls the flow of hydraulics to the joint which causes a change in torque. This digital servo runs at $1 \mathrm{Khz}$. At low limb movement velocities the controller can easily maintain the desired torque. Figure 3 shows the actual torque of the hip joint as the limb is manually moved slowly through its movement range. It can be seen that the torque stays very close to zero. But when the velocity of the limb is higher (Figure 4) it is more difficult for the controller to maintain the desired torque. The valve does not move the hydraulic oil out of the way fast enough. In Figure 4 the limb is being moved quickly by an external force. Throughout this paper the humanoid robot is held at the pelvis in a test stand and a limb position of 0.0 radians is straight down, positive movement is forward, and negative movement is backwards.

We are exploring ways to improve joint torque control further. All hydraulically actuated joints in our robot contain a linear actuator and operate under the same principle. The linear actuator applies a force to a moment arm and the length of the moment arm determines the amount of torque applied to the limb. In the simplest configuration there is a force sensor in series with the actuator and the moment arm changes with angle.

Figures 5 and 6 show the effect of the valve command on joint torque and velocity at various frequencies. The "model"
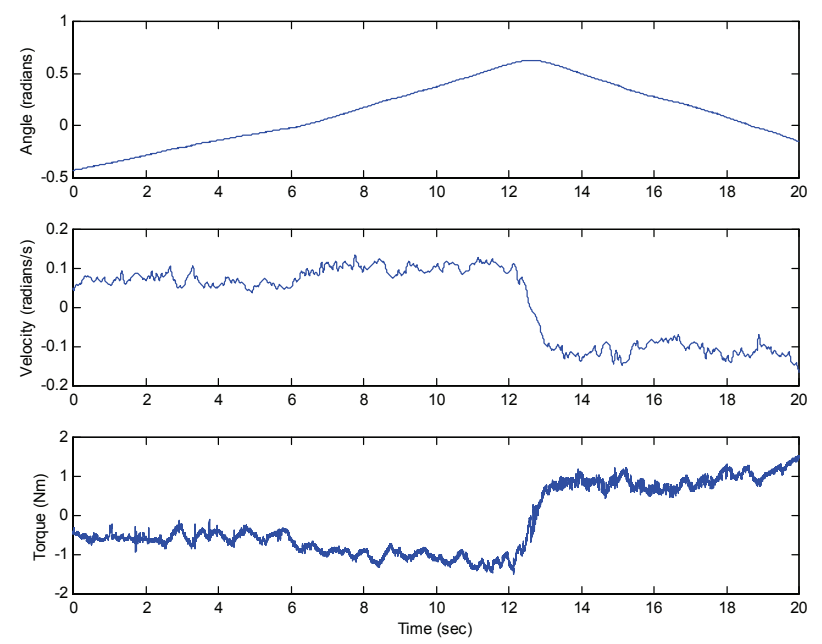

Fig. 3. Joint position, velocity, and torque while the limb is slowly moved by an external force.
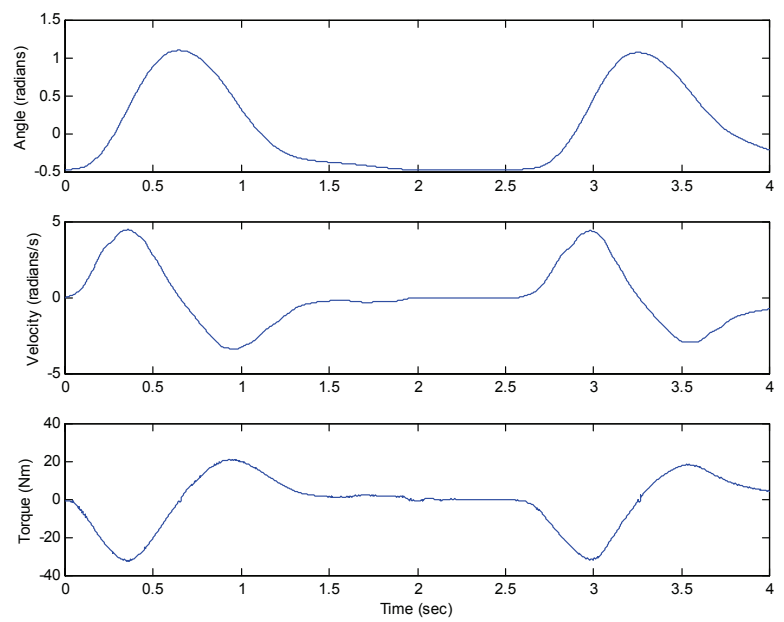

Fig. 4. Joint position, velocity, and torque while the limb is quickly moved by an external force.

trace shown in these graphs is discussed later in this section. The graphs show there is a marked increase in the valve command's effect at around $5 \mathrm{hz}$.

A linearized discrete time model of the state, $\mathbf{x}$, of the system will take the following form:

$$
\mathbf{x}_{t+1}=\mathbf{x}_{t} \cdot \mathbf{A}+u \cdot \mathbf{B}
$$

where

$$
\mathbf{x}=[\theta, \dot{\theta}, \tau]
$$

$$
\mathbf{A}=\left[\begin{array}{ccc}
1 & T & 0 \\
M \cdot g \cdot \ell \cdot T & 1-d 1 \cdot T & \frac{T}{I} \\
\hline I & -a^{2}(\theta) \cdot d 3 \cdot T & 1-d 2 \cdot T
\end{array}\right]
$$



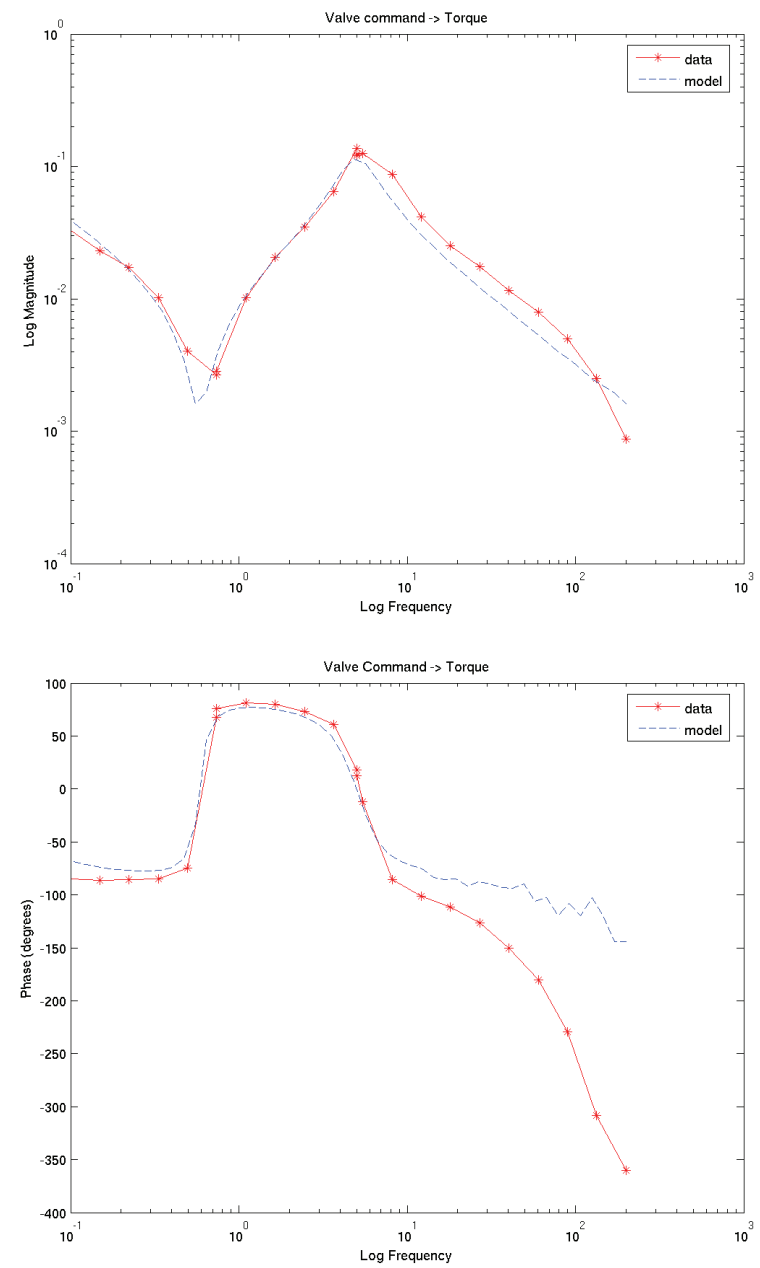

Fig. 5. Bode plots of the effect of the valve command on torque magnitude (top) and torque phase (bottom).

$$
\mathbf{B}=\left[\begin{array}{lll}
0 & 0 & a(\theta) \cdot k \cdot T
\end{array}\right]
$$

and the control input $u$ is the valve command. For the variables in $\mathbf{A}$ and $\mathbf{B}$; $T$ is the sampling interval, $M$ is the mass of the limb, $g$ is $9.81 \mathrm{~m} / \mathrm{s}^{2}, \boldsymbol{\ell}$ is the distance of the center of mass from the pivot point, $I$ is the moment of inertia of the limb about the pivot point, $a(\theta)$ is the moment arm, $d 1$ is the viscous friction, and $k$ is a conversion of valve inputs to torque. There are two parameters that define the actuator dynamics: $d 2$ is the leakage within the actuator from one side of the piston to the other, and $d 3$ relates the velocity to the change in actuator force. From experimental data we identified the values of this model at $\theta=0.5$ to be as follows:

$$
\begin{gathered}
\mathbf{A}=\left[\begin{array}{ccc}
1 & T & 0 \\
-0.01348 & 0.9993 & 0.0002353 \\
0 & -4.0985 & 0.98229
\end{array}\right] \\
\mathbf{B}=\left[\begin{array}{lll}
0 & 0 & 0.000198226
\end{array}\right]
\end{gathered}
$$
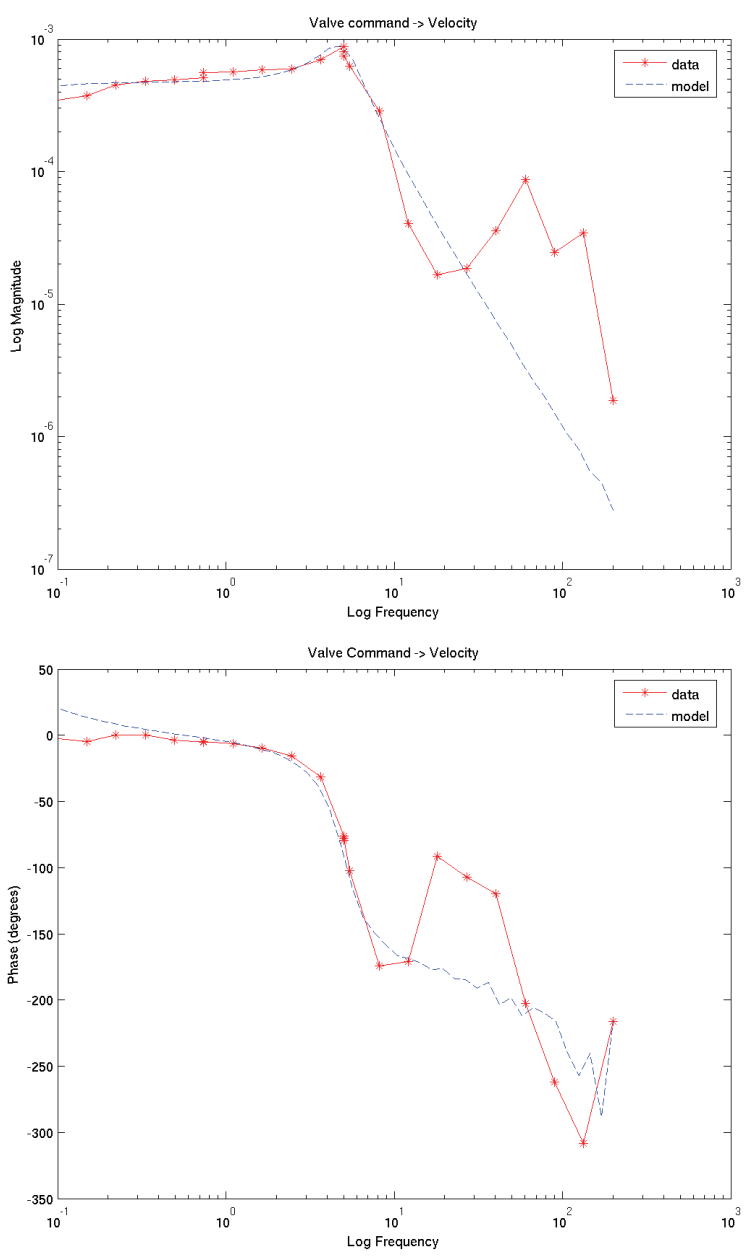

Fig. 6. Bode plots of the effect of the valve command on joint velocity magnitude (top) and joint velocity phase (bottom).

The "model" trace in Figures 5 and 6 shows the result of this model compared to the data. The model fits the data very well to about 10hz. These results show that we have a reasonable understanding of the operation of this joint at low frequencies.

\section{iII. Controller Testing}

We created a variety of controllers and performed tests on the joint to demonstrate the stability of our controllers and to further verify our understanding of its operation. In this section we will present the results of the controllers that we created for compensating for gravity and the actuator dynamics, and in the next section we will present step responses.

\section{A. Gravity Compensation}

In the gravity compensation test, the joint torque is set to support the weight of the leg. The model needed to generate gravity compensation was created by collecting data from the robot while the leg was placed in a variety of positions and various amounts of external torque was applied. The data was fit to a model that included the external torque and the weight of the leg. To obtain the desired torque on the leg a 


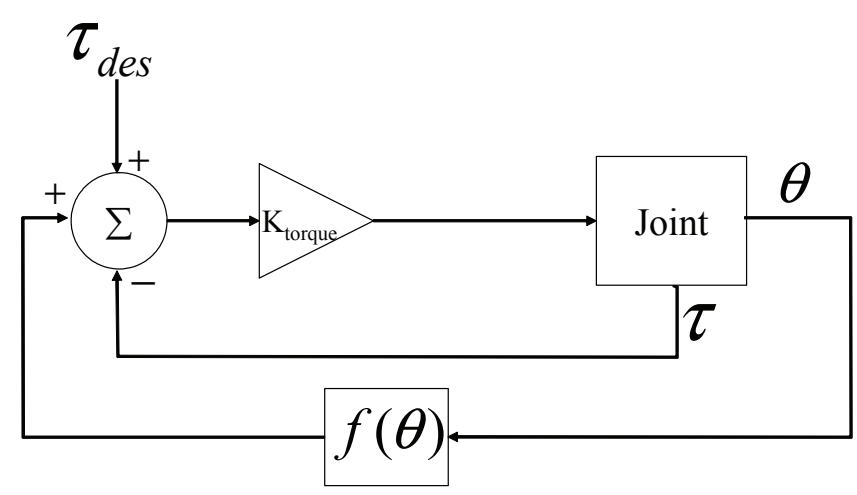

Fig. 7. A torque controller that includes gravity compensation. $f(\theta)$ computes the torque needed to support the weight of the limb for a given limb position $\theta$.

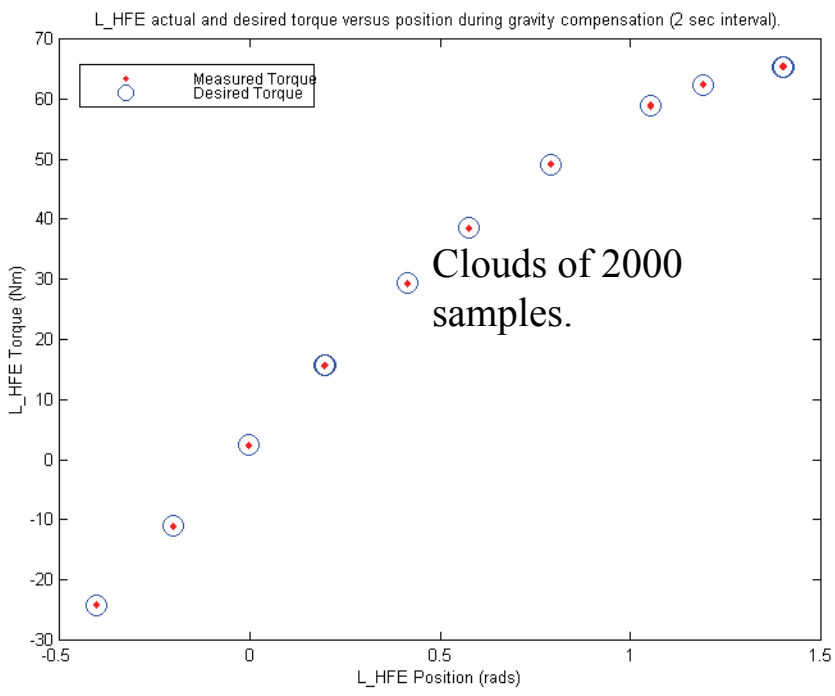

Fig. 8. Actual and desired torque at various positions for 2-second intervals. The desired torque is the compute amount of torque needed to support the weight of the limb.

high gain torque loop was used. Figure 7 shows the gravity compensation controller. For the results presented, $\tau_{\text {des }}$ is set to zero and $K_{\text {torque }}$ is set to 90 .

Figure 8 shows the desired and actual torque during twosecond intervals when the leg is placed at a variety of positions. Even though the trials in the graph appear to be a single dot, each is actually a cloud of 2000 samples during the 2 -second interval. It can be seen that the leg remains almost motionless when placed in a variety of positions.

\section{B. Compensation for the Actuator Dynamics}

The graph in Figure 9 shows that even if the desired torque is 0 when the limb is swung as a pedulum, the limb does not swing freely and quickly damps out even with the use of the high gain torque servo and desired torque set to zero. To compensate for the valve not keeping up with the velocity of the limb, compensation for actuator dynamics
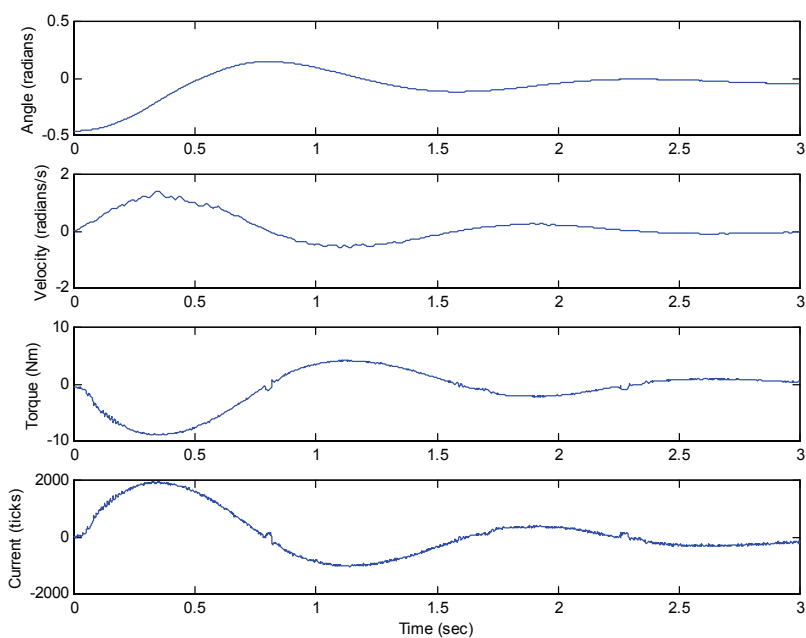

Fig. 9. The velocity, torque, and valve command when the limb is dropped from about -0.45 radians. The joint is running the servo shown in Figure 2 with the desired torque is set to 0 .

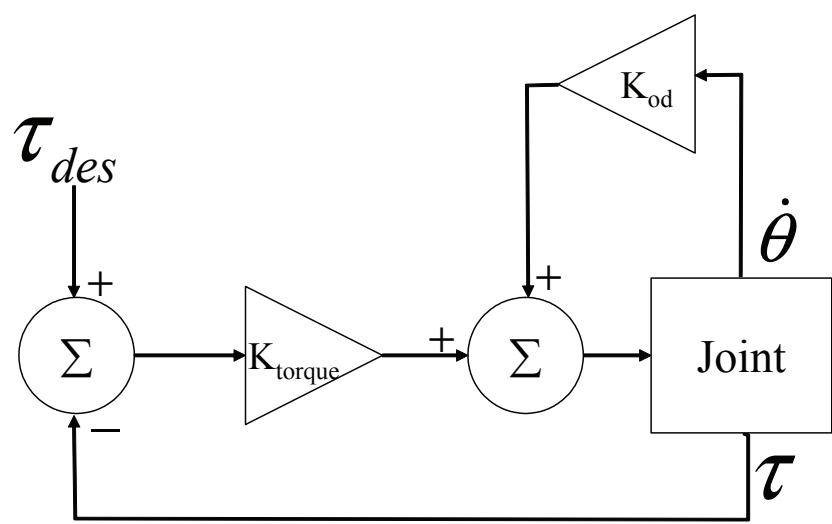

Fig. 10. A torque controller with oil damping included to provide a feedforward command to compensate for slow valve response at faster movements.

was incorporated. Figure 9 shows the valve current while the controller is trying to maintain the desired torque when the leg is released from -0.45 radians.

To make the controller more effective, we provide a feedforward valve command in proportion to the velocity of the limb. The parameters for control of the feed-forward valve current can be determined from the data shown in Figure 9 to fit a simplified model: $\mathrm{V}_{F F}=\dot{\theta} * K_{o d}$. In this simplified model there is only one unknown parameter, the actuator dynamics compensation gain, $K_{o d}$. Even though this model is not entirely correct due to the changing moment arm, it is effective at reducing torque during faster moves. The simplified feed forward controller is shown in Figure 10. For the results presented, $\tau_{\text {des }}$ is set to zero. Figure 11 shows the performance of this controller for 3 seconds to compare with the results shown in Figure 9. Figure 12 shows the performance of the controller during a 20 second interval. We intentionally set the gain of $K_{o d}=1700$, lower than the 

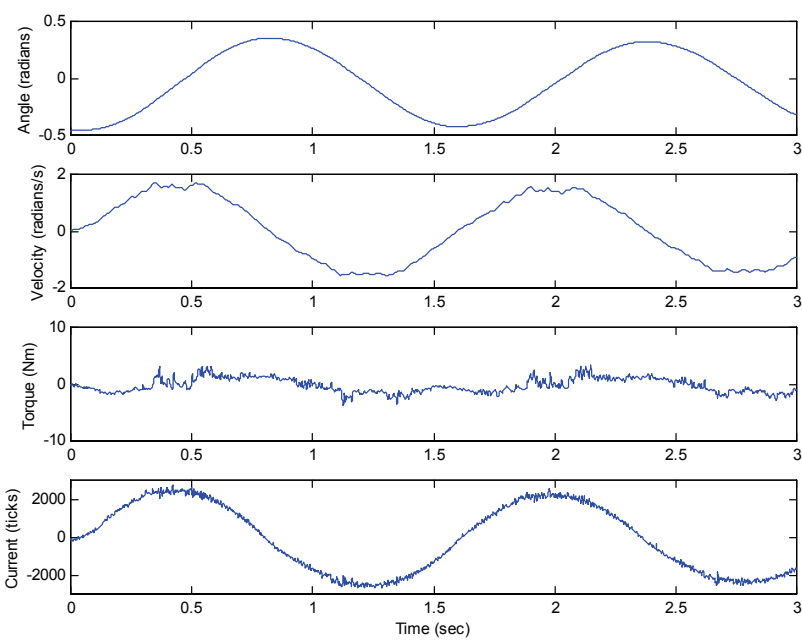

Fig. 11. The results of using the controller shown in Figure 10. The torque is lower compared to Figure 9 where actuator dynamics compensation is not used.
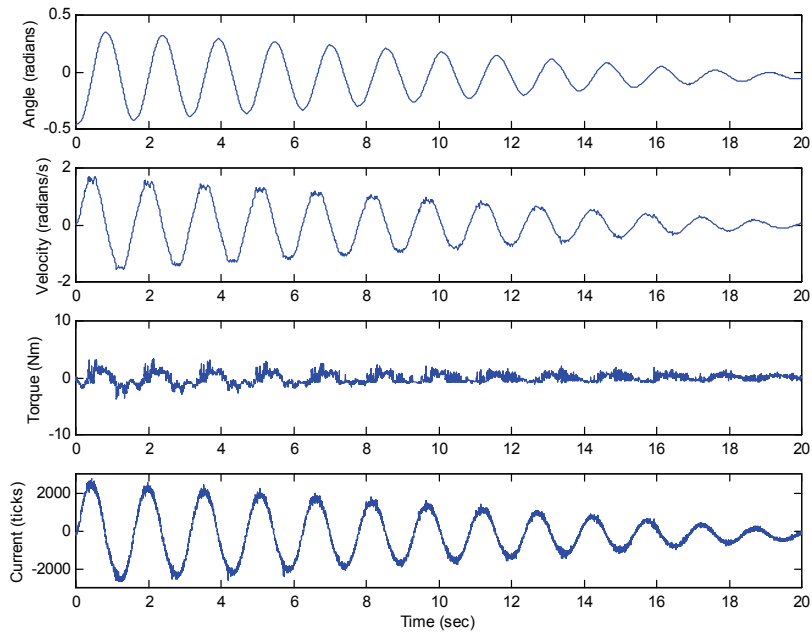

Fig. 12. The results of using the controller shown in Figure 10.

computed optimum of 1760 for safety and it can be seen that the energy in the leg eventually damps out.

\section{Gravity Plus Actuator Dynamics Compensation}

The previous two sections show that gravity and actuator dynamics compensation can help to compensate for the limb's weight and the actuator dynamics. When gravity compensation is used by itself, the limb remains motionless when placed in a position. If the limb is pushed by an external force, the limb quickly comes to rest at a new location due to the actuator dynamics. Since the actuator dynamics compensation removes the damping effect of the actuator dynamics, if it is combined with gravity compensation, a push of the limb would impart energy into the limb and the limb would continue to move at a constant velocity. The controller that combines gravity and

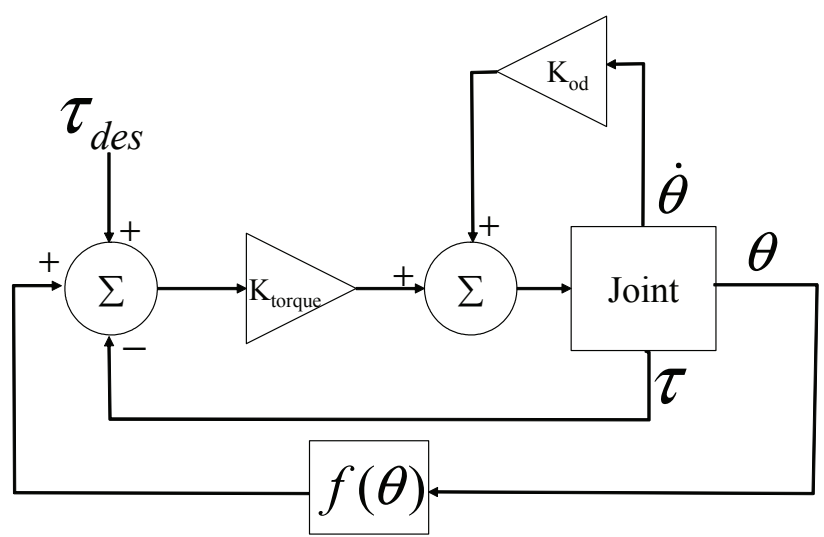

Fig. 13. A controller that combines gravity compensation and oil damping.
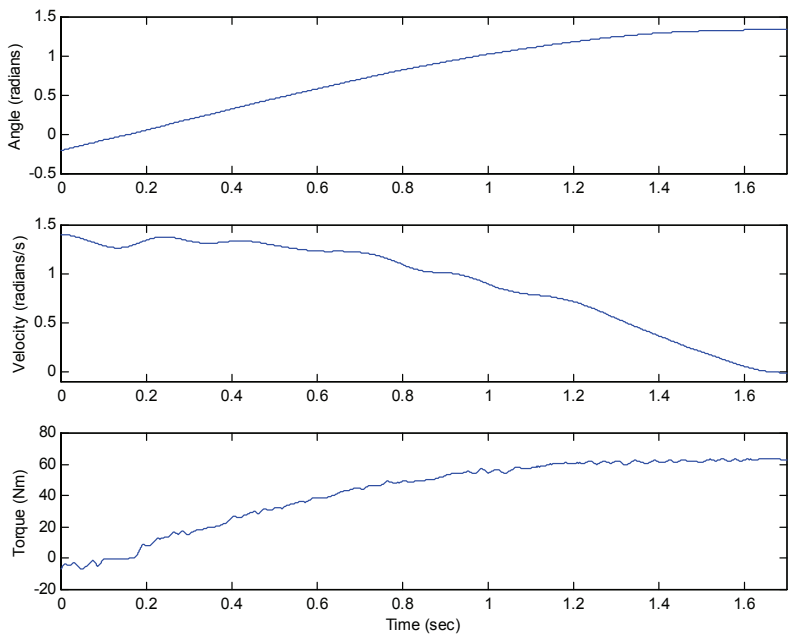

Fig. 14. The movement of the limb when it is pushed forward while running the controller shown in Figure 13.

actuator dynamics compensation is shown if Figure 13. This controller was tested on the limb with $\tau_{\text {des }}$ set to 0 .

Figures 14 and 15 show the performance of this controller when the limb is pushed by an external force. The graphs show the movement of the limb after the external force is removed. In Figure 14 a forward push is applied. Notice the velocity damps out due to $K_{o d}$ being set below the optimum value. This figure also shows the changing torque due to the gravity compensation loop. In Figure 15 the limb is given a forward push. The limb hits the mechanical stop, a metal-tometal contact, at -0.464 radians and bounces back cleanly. These results demonstrate that the gravity compensation and oil compensation controllers work together well and do not induce instabilities.

\section{TORQue CONTROL}

Figure 16 shows the controller we created that allows for a desired position, velocity, and feed-forward torque. The output of this controller is fed to the controller shown in Figure 13. By varying the position gain, $K_{\text {pos }}$, and velocity gain, $K_{v e l}$, 

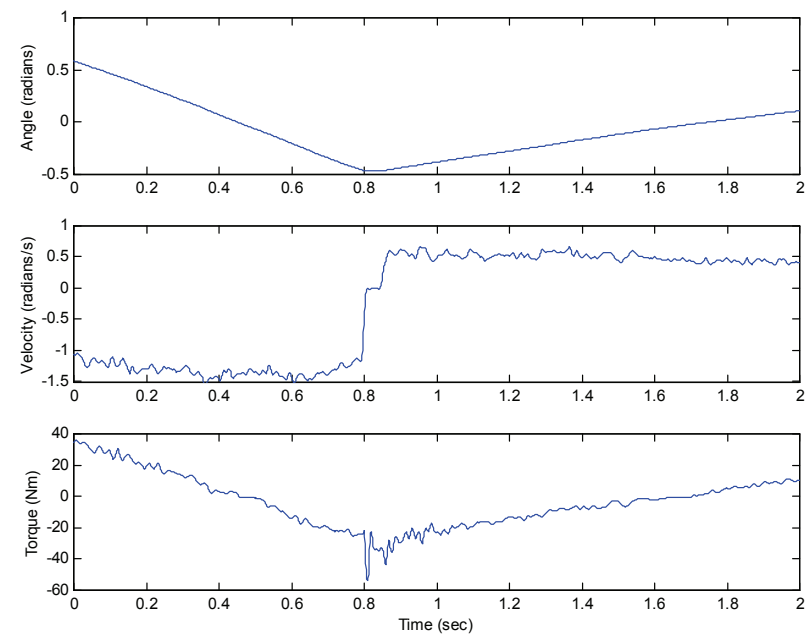

Fig. 15. The movement of the limb when it is pushed backward while running the controller shown in Figure 13. The limb hits a hard stop at -0.464 radians and bounces back.

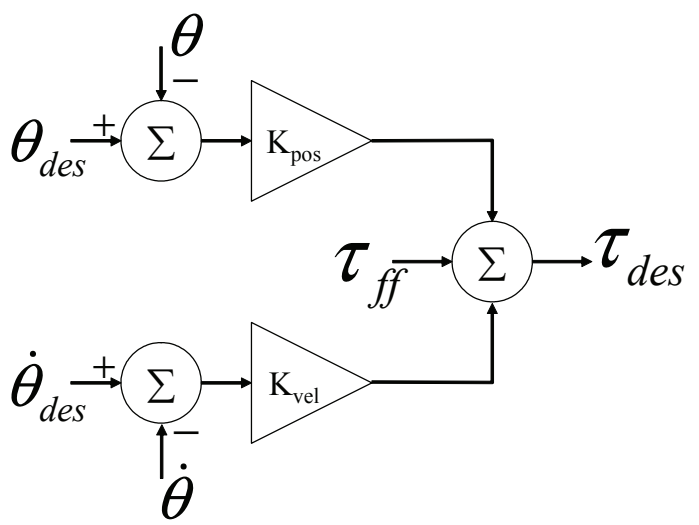

Fig. 16. The torque controller that computes the desired torque from desired positition, $\theta_{d e s}$, and velocity, $\dot{\theta}_{d e s}$. A feed-foward torque, $\tau_{f f}$, can also be specified.

this controller can take on a variety of characteristics. With the gains set to 0 , the limb will be extremely compliant as shown above. Increasing the position and velocity gains will increase the stiffness of the limb. Figure 17 shows the position of the limb while an external torque is applied. During this trial the position and velocity gains are set to be very high. It can be seen that there is very little movement of the limb for large external forces. Rotation stiffness is the applied torque, $\tau$, divided by the rotational displacement, $\theta$. With the gains set to zero, a small torque applied to the limb creates a large displacement resulting in a stiffness of near zero. When the gains are set to high values the stiffness computed from the data in Figure 17 is $4600 \mathrm{Nm} /$ radian.

To demonstrate the ability of this controller to move the limb when it is stiff, we performed a step response test. In this test the desired position of the limb is instantanously changed by 0.1 radians while the desired velocity and feed-forward
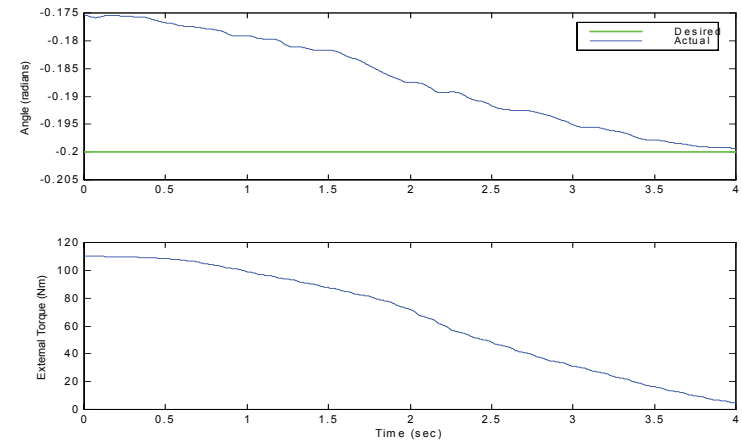

Fig. 17. The position of the limb while an external torque is applied to the limb. The limb is held in place by the controller shown in Figure 16 with the position and velocity gains set very high.
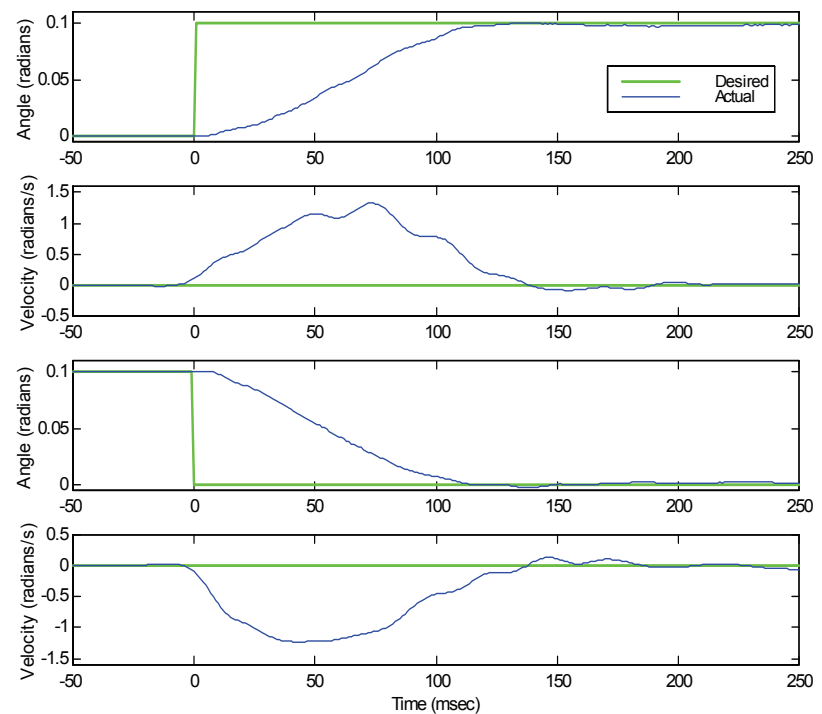

Fig. 18. Step maneuvers performed while using the controllers shown in Figures 13 and 16. Position and velocity gains are high to make the limb stiff.

torque are held at 0 . Figure 18 shows the result of performing this test while moving the limb forward and backward. The position and velocity gains are set to be very high during this test $\left(K_{\text {pos }}=4500\right.$ and $\left.K_{\text {vel }}=250\right)$. The results show that the controller can make this movement in just over $100 \mathrm{~ms}$ and stably rests at the desired position.

If gravity and actuator dynamics compensation were perfect, a desired torque other than zero would move the limb according to the function $\tau=I \cdot \ddot{\theta}$. If the inertia, $I$, is known, a trajectory that includes acceleration, $\ddot{\theta}$, can be used to compute a feed-forward torque that will move the limb as needed. From the collected data we estimated the limb to have an inertia of $4.25 \mathrm{kgm}^{2}$. We created fifth-order trajectories for the limb to move from one position to another. The starting and ending velocities and accelerations of the trajectories are set to zero. Fitting the trajectories to a fifth-order equation 

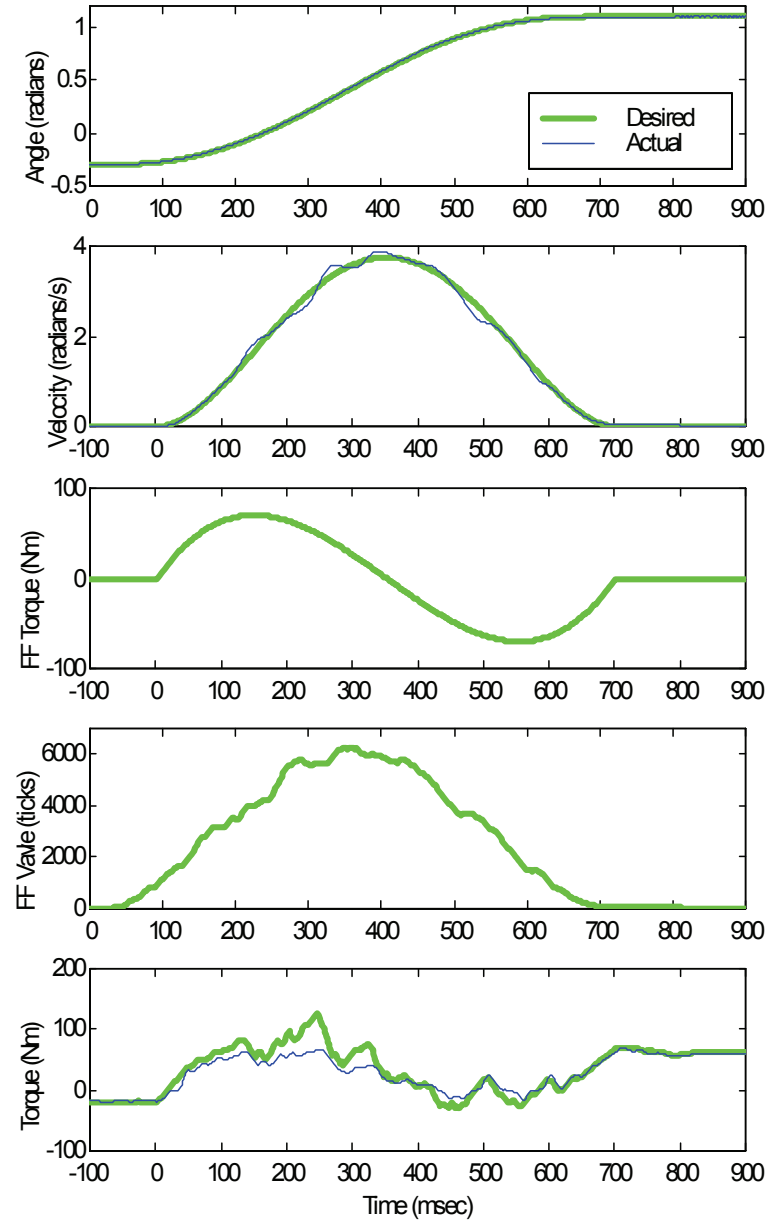

Fig. 19. A fifth-order trajectory ran using the controller shown in Figure 16. The feed-forward valve command is generated by the actuator dynamics compensator. The feed-forward torque is computed from the desired acceleration and the inertia of the limb.

ensures smooth positions, velocities, and accelerations during the trajectories. We used the acceleration to compute a feedforward torque at each time step. Figure 19 shows the result of following a trajectory between -0.3 and 1.1 radians. The feedforward valve command is generated by the actuator dynamics compensator. The feed-forward torque is computed from the desired acceleration and the inertia of the limb. It can be seen that the desired torque is different from the feed-forward torque due to the input from the gravity compensator and the effect of the controller using high position and velocity gains.

\section{Discussion AND CONCLUSION}

Our results show that hydraulic actuators can satisfy humanoid robot actuation requirements. An advantage of hydraulic actuation is that the actuators are small relative to the power they provide. This allows limbs to be strong and light weight. But there are also disadvantages to hydraulic systems. The oil must be routed to each actuator through hydraulic hoses. If the hoses are not large enough, speed and strength be limited. There must also exist a pump to supply the high pressure oil. In our case the pump is not portable and is connected to the robot via two hoses. Other systems use portable pumps with potentially less capacity. Another disadvantage of this system is that some of the actuators leak a small amount of oil. Future technologies are working toward minimizing these disadvantages such as creating small and powerful hydraulic pumps that can be carried on the robot.

We have created a variety of controllers that satisfy constraints from very compliant to very stiff. We have shown that our limb can be so compliant that it will move with a very slight touch and can also be extremely stiff so as to move verly little when even a large force is applied. We have also shown that the limb is stable when running our controllers. The close fit of the numerical model to the actual data along with the success of our controllers are an indication that we understand the operation of this joint. We will continue to design controllers for the other joints of our humanoid robot. We plan to investigate and implement controllers that operate over multiple joints.

\section{ACKNOWLEDGMENT}

This material is based upon work supported in part by the National Science Foundation under grants CNS-0224419, ECS-0325383, and EEC-0540865.

\section{REFERENCES}

[1] V. Hayward, "Design of hydraulic robot shoulder based on combinatorial mechanism," in Experimental Robotics 3, T. Yoshikaswa and F. Myiazaki, Eds. Springer Verlag, 1994, pp. 297-310.

[2] M. Namvar and F. Aghili, "A combined scheme for identification and robust torque control of hydraulic actuators," Journal of Dynamic Systems, Measurement, and Control, vol. 125, pp. 595-606, 2003.

[3] A. D. Luca, A. Albu-Schaffer, S. Haddadin, and G. Hirzinger, "Collision detection and safe reaction with the dlr-iii lightweight manipulator arm," in 2006 IEEE/RSJ International Conference on Intelligent Robots and Systems, Bejing, China, October 2006, pp. 1623-1630.

[4] G. Palli, C. Melchiorri, T. Wimbock, M. Grebensein, and G. Hirzinger, "Feedback linearization and simultaneous stiffness-position control of robots with antagonistic joints," in 2007 IEEE/RSJ International Conference on Robotics and Automation, Rome, Italy, April 2007, pp. 1623 1630.

[5] M. A. Jarrah and O. M. Al-Jarrah, "Position control of a robot manipulation using continuous gain scheduling," in IEEE International Conference on Robotics and Automation, 1999, pp. 170-175.

[6] B. Paijmans, W. Symens, H. V. Brussel, and J. Swevers, "Gainscheduling control for mechanics systems with position dependent dynamics," in International Conference on Noise and Vibration Engineering, 1999 , pp. 170-175.

[7] S. Hyon and G.Cheng, "Gravity compensation and full-body balancing for humanoid robots," in 2006 IEEE-RAS International Conference on Humanoid Robotics, 2006, pp. 214-221.

[8] S. Hyon and G. Cheng, "Passivity-based full-body force control for humanoids and application to dynamic balancing and locomotion," in 2006 IEEE/RSJ International Conference on Intelligent Robots and Systems, Bejing, China, October 2006, pp. 4915-4922.

[9] G. Bilodeau and E. Papadopoulos, "Experiments on a high performance hydraulic manipulator joint: Modelling for control," in Proceedings of the 1997 International Symposium on Experimental Robotics (ISER '97), Barcelona, June 1997.

[10] C. haur Wu and R. P. Paul, "Manipulator compliance based on joint torque control," in 19th IEEE Conference on Decision and Control, vol. 19, 1980, pp. 88-94.

[11] M. Zinn, O. Khatib, B. Roth, and J. K. Salisbury, "Towards a humancentered intrinsically-safe robotic manipulator," in IEEE Robotics and Automation Magazine, vol. 11, no. 2, June 2004, pp. 12-21. 